\title{
Género y Nivel Socioeconómico de los Niños: Expectativas del Docente en Formación
}

\section{Gender and Socioeconomic Status of Children: Pre-Service Teachers' Expectations}

\author{
M. Francisca del Río y Jaime Balladares \\ Universidad Diego Portales
}

\begin{abstract}
Se puso a prueba empírica el efecto del género y nivel socioeconómico (NSE) de los alumnos en las expectativas de los profesores en formación. Participaron 108 estudiantes de pedagogía, los que leyeron una historia acerca de un estudiante hipotético que presentaba problemas conductuales y de rendimiento. Se manipuló el sexo y NSE del estudiante, creando 4 condiciones. Los participantes percibieron que los estudiantes de NSE bajo tenían peores características personales y un futuro académico menos promisorio, no encontrándose diferencias por género. Los participantes de último año de carrera presentaron expectativas más positivas para los niños de NSE bajo que los de primer año. Las expectativas más negativas de los estudiantes de pedagogía respecto de los estudiantes de NSE bajo podrían contribuir a explicar la baja eficacia de los profesores en escuelas vulnerables.
\end{abstract}

Palabras clave: educación inicial, eficacia docente, expectativas, creencias

The effect of student gender and socioeconomic status (SES) on pre-service teachers' expectations of students was examined empirically. Participants were 108 pre-service teachers who read a scenario about a hypothetical student with academic and behavioral challenges. The gender and SES of the student were varied to create 4 conditions. Pre-service teachers perceived that low SES students have less promising futures and more negative personal characteristics, and demonstrated no differences about gender. Senior students had more optimistic expectations for low SES children than freshmen students did. Findings suggest that pre-service teachers are likely to develop negative expectations toward low SES students. These preconceived attitudes may help explain why teacher efficacy tends to be lower in economically disadvantaged schools.

Keywords: pre-service teachers, teacher efficacy, beliefs, expectations

\section{Antecedentes Teóricos}

Una de las metas del sistema educacional chileno es entregar un ambiente educativo de calidad, donde todos los alumnos puedan alcanzar su potencial académico. Pero la realidad muestra que, desgraciadamente, esto no sucede así.

En Chile existen diferencias notorias en los resultados académicos de los niños, principalmente de acuerdo a dos factores, el nivel socioeconómico (NSE) y el género. Esto se refleja en el perfil que Chile presenta en las pruebas de logro académico, tanto a nivel nacional como internacional. En efecto, mientras que en la mayoría de los países no se presentan diferencias de rendimiento en- tre hombres y mujeres en la prueba PISA (medición internacional) y, entre los países que las presentan predominan aquellos en que las mujeres obtienen puntajes más altos, los resultados de Chile sobresalen por las amplias brechas de género en el logro académico a favor de los hombres.

En detalle, en la prueba PISA Chile mostró las diferencias más grandes a favor de los estudiantes varones en ciencia y matemática y, la más pequeña, a favor de las mujeres en lectura (MIDE UC, 2008). Asimismo, esta evaluación mostró que Chile es uno de los países en que el NSE tiene una mayor incidencia en los resultados, de modo que la brecha entre los estudiantes que tienen peores y mejores condiciones socioeco-

M. Francisca del Río y Jaime Balladares, Facultad de Educación, Universidad Diego Portales, Santiago, Chile.

La correspondencia relativa a este artículo debe ser dirigida a M. Francisca del Río, Facultad de Educación, Universidad Diego Portales, Vergara 210, Santiago, Chile. E-mail: francisca.delrio@mail.udp.cl 
nómicas es muy amplia y siempre a favor de los niños con mayores recursos (Chile, Ministerio de Educación, 2007). Estas brechas se repiten en mediciones de logro académico nacionales, tales como el Sistema de Medición de Calidad de la Educación (SIMCE). Así, en las últimas tres mediciones de las que se conocen los resultados (años 2007, 2008 y 2009), se observa que tanto en los niveles de $4^{\circ}$ y $8^{\circ}$ básico como en $2^{\circ}$ medio -y en todos los subsectores del conocimiento evaluados- los resultados están fuertemente asociados al NSE: a mayor NSE los estudiantes obtienen puntajes promedio más altos. Por otra parte, al mirar estos resultados desde una perspectiva de género, estos reflejan que, en general, las mujeres obtienen mejores puntajes en las pruebas de Lenguaje y Comunicación, mientras que los hombres sobresalen en Ciencias y Matemática (SIMCE, 2008, 2009, 2010).

¿Cómo se explican estas diferencias en un sistema educativo que busca entregar igual calidad de educación a todos los niños?

Más allá de las clásicas explicaciones que en Chile se barajan para entender el menor rendimiento de los niños de NSE bajo -atribuyéndolo a la menor calidad de la educación pública- el origen de las diferencias antes mencionadas aún no está totalmente esclarecido. Pero es razonable pensar que la respuesta podría residir tanto en los eventos que ocurren dentro de la sala de clases como en las creencias, estereotipos y teorías implícitas de los individuos involucrados en el proceso de enseñanza/aprendizaje. Respecto a este último grupo de causas, la literatura señala que la exposición repetida a casos de estudiantes de bajo NSE y de género femenino de bajo rendimiento puede causar que los profesores desarrollen expectativas acerca del potencial de los estudiantes, basadas en su género y NSE. Estas expectativas pueden, a su vez, afectar el sentido de responsabilidad y de autoeficacia de los profesores (Mistry, White, Benner \& Huynh, 2009; Rashid, 2009; Warren, 2002). Así, si los profesores sostienen la creencia de que los logros académicos de los estudiantes están determinados por factores más allá de su control (por ejemplo, NSE y género), mostrarán una baja motivación para encon- trar maneras de mejorar el logro de estos estudiantes (Diamond, Randolph \& Spillane, 2004). De esta forma, los estudiantes que presentan el mayor riesgo de ser estereotipados negativamente en lo académico son también los más vulnerables de cargar con bajas expectativas de sus profesores (Jussim \& Harber, 2005).

Esta dinámica también se ha observado en profesores en formación. Ladd y Linderholm (2008) llevaron a cabo una investigación en la que a estudiantes de pedagogía se les entregó la descripción de alumnos asignados aleatoriamente en distintos grupos de rendimiento académico. Una vez que los estudiantes de pedagogía ya conocían el rendimiento asignado a cada grupo, se les pidió describir las características de las conductas de cada uno de ellos (aun agrupados por rendimiento), a través de la observación de un video de su comportamiento en clases. Los resultados mostraron que, de manera sistemática, los profesores en formación tipificaron como más negativas las conductas de los alumnos que de manera azarosa fueron incluidos en los grupos de menor rendimiento. Esto demuestra que ya desde antes de ejercer como profesores los estudiantes de pedagogía están predispuestos a tener expectativas acerca de sus alumnos y a actuar en concordancia con ellas. Otro estudio también mostró que los profesores en formación presentan prejuicios frente a ciertos grupos sociales, como los inmigrantes (Navas \& Sánchez, 2010).

La presente investigación tuvo como objetivo conocer si este fenómeno estaba presente entre los estudiantes de pedagogía en Chile. Más específicamente, la pregunta que guió esta investigación fue si los profesores en formación tienen expectativas diferenciadas para sus futuros estudiantes dependiendo del NSE y género de los niños. Consecuentemente, se examinaron empíricamente las expectativas que los estudiantes de pedagogía tienen acerca de sus futuros estudiantes, manipulando de manera sistemática las variables género y NSE de un estudiante hipotético. Los hallazgos permitirían llevar a cabo intervenciones durante la formación pedagógica, con el fin de que los profesores comiencen su vida profesional ya conscientes de sus creencias y ex- 
pectativas y de las consecuencias que ellas pudieran tener en el trato con sus alumnos $y$, por ende, en su rendimiento académico.

\section{Expectativas: NSE y Género}

A partir del reconocido estudio de Rosenthal y Jacobson (1966), que fue pionero en demostrar el efecto de las expectativas de los profesores sobre el logro académico de sus alumnos, numerosas investigaciones han reeditado este efecto, asociándolo con factores distintivos de los estudiantes. Entre estas, algunas han demostrado que las expectativas educativas de los profesores pueden verse afectadas por el NSE de sus alumnos, lo que a su vez se refleja en prácticas educativas sesgadas. Por ejemplo, tanto Mistry et al. (2009) como Dusek y Joseph (1983) reportan que los profesores esperan un menor rendimiento de sus alumnos de NSE bajo que de los de mejor situación socioeconómica y que esto hace que traten a sus estudiantes de manera diferenciada (en concordancia con sus creencias). Por otra parte, las investigaciones que han abordado las temáticas de género y educación han demostrado que, pese a los esfuerzos que tienden hacia la igualdad de oportunidades y equidad entre mujeres y hombres, los educadores siguen tratando a sus alumnos y alumnas de manera distinta. Así, la literatura afirma que la mayoría de los profesores cree tratar a hombres y mujeres de manera equitativa, pero que esto rara vez ocurre en la práctica (Gray \& Leith, 2004). Esto último lo demuestran los estudios de Derman-Sparks y The A.B.C. Task Force (1989), que sugieren que los profesores tienden a recompensar más a las niñas por su apariencia, su nivel de cooperación y su obediencia, mientras que a los niños los premian por sus logros.

Múltiples investigaciones han dado cuenta de la importancia que tienen las expectativas de los profesores acerca de las capacidades académicas de sus alumnos, dado que estas se traducen en una forma diferenciada de actuar y enseñarles y, por lo tanto, tienen también un impacto en su rendimiento académico (Auwarter \& Aruguete, 2008; Palardy \& Rumberger, 2008). A pesar de lo anterior, casi no existen a nivel nacional es- tudios que den cuenta de esta dinámica.

Como una manera de subsanar esta carencia, en la presente investigación se puso a prueba de manera empírica la afirmación de que el género y el NSE de los estudiantes tienen una influencia en las expectativas de los profesores en formación, formulando la hipótesis de que las evaluaciones de los estudiantes de sexo femenino y NSE bajo: (a) presentan características académicas personales más negativas, (b) muestran una mayor necesidad de apoyo académico y (c) se espera de ellos un futuro académico menos promisorio, en comparación con los otros grupos de estudiantes. Por último, también se postula que no deben presentarse diferencias significativas entre las expectativas de los profesores en formación que cursan el primer año de su carrera y las de los que están terminando sus estudios.

\section{Método}

\section{Diseño}

La investigación utilizó un diseño de comparación entre grupos, presentándose a estudiantes de pedagogía uno de cuatro escenarios hipotéticos, en los cuales un estudiante variaba sistemáticamente respecto de su género y NSE, mientras que todas sus características adicionales se mantenían constantes. Los participantes fueron asignados aleatoriamente a una de las cuatro condiciones experimentales, las que se señalan más adelante.

\section{Participantes}

Se utilizó una muestra intencionada de estudiantes de pedagogía. Participaron 108 alumnos de las carreras de pedagogía de la Facultad de Educación de una universidad de carácter privado, ubicada en la Región Metropolitana, el 93\% de ellos de sexo femenino $(n=101)$. Sesenta y siete $(62 \%)$ de ellos estaban cursando el primer año de la carrera, mientras que 41 (38\%) cursaban el último año de estudios ( $4^{\circ}$ año). La muestra incluyó a 50 estudiantes de la carrera de pedagogía en educación básica (46\%) y 58 de la carrera de educación parvularia (54\%). Se optó por incluir en la muestra de manera 
indiferenciada a los estudiantes de ambas carreras, a pesar de los diferentes contextos en que se encuadrará su labor -jardines infantiles o escuelas básicas-, pues todos ellos tendrán que enseñarles a niños y en ambas situaciones estarán presentes sus expectativas y sus potenciales efectos.

\section{Instrumento}

A cada participante se le solicitó leer la historia de un estudiante con aparentes problemas académicos y conductuales, la que fue traducida y adaptada del instrumento utilizado en la investigación de Auwarter y Aruguete (2008).

La manipulación experimental consistió en variar sistemáticamente el género y NSE del estudiante protagonista de la historia, de manera de producir cuatro condiciones: (a) NSE bajo-niña, (b) NSE altoniña, (c) NSE bajo-niño y (d) NSE alto-niño. La variable género variaba a través del uso diferenciado de pronombres y nombres que indicaran el sexo del estudiante protagonista de la historia. El NSE se modificó alterando las profesiones de los padres del estudiante (madre médico y padre abogado versus madre trabajadora de casa particular y padre cesante), la dependencia del establecimiento educacional al que asistía (municipal versus particular) y la ubicación del mismo (Pudahuel versus Las Condes).

Solo la información que se puede observar entre paréntesis variaba entre las condiciones:

(Marcela, Marcelo) es una (niña, niño) que asiste a una escuela (municipal, particular) de (Pudahuel, Las Condes). (Marcela, Marcelo) vive con sus padres y es la/el segunda/o hermana/o de tres. Su madre es (trabajadora de casa particular, médico) y su padre está/es (actualmente cesante, abogado). Ella/él tiene un nivel de inteligencia promedio, pero en matemáticas se está sacando malas notas. Además, no está cumpliendo con sus tareas y trabajos en varios ramos y no ocupa de manera eficiente su tiempo en clases. Hace un tiempo, (Marcela, Marcelo) solía tener una actitud positiva hacia la escuela, tenía buenas notas y era evaluada/o de manera positiva por sus profesores. Pero en las últimas semanas ha faltado repetidamente a clases y ha recibido varias anotaciones por mala conducta. Por ejemplo, ha sido agresiva/o con sus compañeros, verbal y físicamente, situación que se repite por lo menos una vez por semana. Sus padres han mantenido reuniones con el profesor de (Marcela, Marcelo) pero la situación no mejora.

Luego de leer la historia, se solicitó a los participantes ponerse en el lugar del profesor del niño protagonista de la historia para contestar un cuestionario. Este cuestionario también fue adaptado del utilizado en la investigación de Auwarter y Aruguete (2008).

El cuestionario a responder contenía cuatro escalas dirigidas a evaluar:

1. Las características personales del estudiante $(C P)$ : (alfa de Cronbach $\left.=0,75^{1}\right)$. Consiste en 15 preguntas con una escala de diferencial semántico de cinco puntos, en la que el mayor valor se corresponde con rasgos más positivos (ejemplo: "Evalúe al estudiante en las siguientes características: incompetente-competente").

2. La necesidad de apoyo académico adicional $(A A A)$ : (alfa de Cronbach = 0,60). Consiste en cinco afirmaciones en las que se debe señalar el grado de acuerdo con cada una de ellas, en base a una escala Likert de cinco puntos, reflejando un menor valor una mayor necesidad académica adicional (ejemplo: se necesita una intervención especial con este estudiante).

3. Las expectativas acerca del futuro del estudiante (EFE): (alfa de Cronbach = 0,83 ). Consiste en seis afirmaciones en las que se debe señalar el grado de acuerdo con cada una de ellas, en base a una escala Likert de cinco puntos, reflejando un mayor valor expectativas más positivas (ejemplo: la probabilidad de que abandone la escuela es alta).

4. La credibilidad de la historia (CH): (alfa de Cronbach $=0,72$ ). Consiste en cuatro afirmaciones dirigidas a evaluar

\footnotetext{
1 Los valores alfa de Cronbach reportados fueron calculados en base a la aplicación del instrumento en este estudio.
} 
la credibilidad de la historia. En cada afirmación se debe señalar el grado de acuerdo en base a una escala Likert de cinco puntos (ejemplo: existen estudiantes reales en la situación de Marcelo/a).

El cuestionario aplicado contenía un último ítem de diferencial semántico de cinco puntos, destinado a señalar el NSE de los personajes protagónicos de las historias (rico a pobre).

\section{Procedimiento}

La realización de este estudio contó con la debida aprobación del comité de ética de la Facultad de Educación participante y con el consentimiento verbal de los estudiantes, a quienes se les explicitaron los objetivos del estudio y la calidad voluntaria de la participación. Los estudiantes fueron evaluados en grupo en una sala especialmente acondicionada para este fin. El cuestionario fue aplicado por los propios investigadores, durante los meses de marzo y abril del año 2009. La aplicación consideró un rango de tiempo entre 20 a 30 minutos.

\section{Calidad de los Datos y Plan de Análisis}

Antes de contrastar las hipótesis formuladas, se examinó que los datos cumplieran con los requisitos necesarios para llevar a cabo pruebas de comparación de medias. Con el fin de comprobar la normalidad de la distribución de los datos, se aplicó la prueba de Kolmogorov-Smirnov a cada una de las variables dependientes, presentando todas ellas una distribución normal. La variable CP obtuvo un resultado en esta prueba de $K S=0,67, p=0,75 ;$ la variable AAA, de $K S$
$=1,17, p=0,12 ; \mathrm{y}$ la variable $\mathrm{EFE}$, de $K S=$ $0,80, p=0,54$.

También se aplicó la prueba de homogeneidad de varianzas de Levene a cada una de las variables dependientes. Ninguno de los test de Levene aplicados fue significativo $(\underline{p}>0,22)$, por lo que no se podría refutar la hipótesis nula de igualdad de varianzas entre los grupos.

Por último, se calculó la correlación lineal de Pearson entre las tres variables dependientes. Los resultados muestran que todas correlacionan de manera significativa entre sí, lo que refleja una alta coherencia de los datos (ver la Tabla 1).

El plan de análisis consistió en comprobar las hipótesis a través de ANOVAs factoriales de 2 (NSE, alto versus bajo) $\mathrm{x}$ 2 (género) para cada una de las variables evaluadas, tanto para el grupo completo como para las submuestras de $1^{\circ}$ y $4^{\circ}$ año de carrera. Por último, se realizó una prueba $t$ de Student para determinar la eventual diferencia de promedios en cada una de las variables medidas, entre los estudiantes de pedagogía de diferente año de carrera $\left(1^{\circ}\right.$ versus $4^{\circ}$ ).

\section{Resultados}

\section{Chequeo de Manipulación}

Se llevaron a cabo dos chequeos de manipulación con el fin de confirmar que los personajes protagonistas de las historias resultaban creíbles y que no existían equívocos en relación a su NSE.

Este chequeo tuvo como propósito conocer si los participantes percibían correctamente el NSE intencionado de los persona-

Tabla 1

Correlación Entre las Variables Dependientes

\begin{tabular}{ccc}
\hline Variables & AAA & EFE \\
\hline CP & 0,44 & 0,46 \\
AAA & & 0,47 \\
\hline
\end{tabular}

$p<0,01$ 
jes de la historia. Por medio de la prueba $t$ de Student se pudo observar que el promedio de quienes contestaron los ítems en las condiciones donde el protagonista era de NSE alto fue significativamente diferente al de los que participaron en las condiciones en las que el personaje se asociaba a un NSE bajo, $t(106)=16,45, p<0,001, d$ $=3,20,95 \%$ IC $[1,78,2,27]$. Asimismo, los protagonistas de NSE alto $(M=4,13, D E=$ $0,56)$ fueron clasificados como más ricos que los del escenario contrario $(M=2,10, D E=$ 0,70). De esta forma, los resultados indicaron que la manipulación fue percibida de la manera como se intencionó.

Respecto del chequeo dirigido a evaluar la $\mathrm{CH}$, los resultados mostraron que, en general, los participantes consideraron las historias creíbles $(M=1,94, D E=0,45)$, Asimismo, los resultados mostraron que no existieron diferencias significativas de credibilidad entre las cuatro historias, $F(3$, 107) $=1,73, p=0,16$.

\section{Resultados del Test}

Las hipótesis formuladas postulaban que los estudiantes de pedagogía evalúan más negativamente las CP de los protagonistas de NSE bajo y de sexo femenino.

Los promedios de las variables evaluadas de acuerdo a los factores NSE y género se pueden observar en la Tabla 2.

$\mathrm{Al}$ considerar la muestra total, la prueba ANOVA mostró que no existiría una interacción significativa entre los factores sexo y NSE en los resultados relativos a las características personales del protagonista de la historia evaluada, $F(3,107)=0,16, p$ $=0,68$. Por su parte, al examinar los efectos simples de cada variable por separado, se pudo observar que el promedio de la variable CP para las historias en las que el protagonista era de NSE alto $(M=2,68, D E=0,36)$ resultó significativamente mayor $(F[1,107]$ $\left.=8,79, p=0,004, \eta^{2}=0,08\right)$ al promedio de la misma en los casos en los que el protagonista era de NSE bajo $(M=2,46, D E=0,39)$, $95 \%$ ICs $[2,58,2,78]$ y $[2,35,2,57]$, respectivamente; mientras que no se observaron diferencias significativas entre los promedios de CP en las historias en las que el protagonista tenía diferente sexo, $F(1,107)=0,02$, $p=0,86$. Esto muestra que respecto de la variable $\mathrm{CP}$, la información parece apoyar parcialmente la hipótesis formulada: los estudiantes no evaluaron más negativamente las características de los personajes de sexo femenino y NSE bajo, pero parecen haberlo hecho para el conjunto de los individuos de NSE bajo, sin discriminar por género.

Una segunda hipótesis postulaba que los estudiantes de pedagogía plantean una mayor necesidad de AAA para los estudiantes de NSE bajo y de género femenino. Los resultados no parecen apoyar tal interacción, $F(3,107)=0,38, p=0,53$. Un análisis de los efectos simples de cada variable mostró que, respecto del género del protagonista de la historia, no existirían diferencias significativas de los promedios de AAA, $F(1,107)=0,23, p=0,62$, pero sí es posible observar diferencias al comparar los promedios de la variable AAA en los casos de diferente NSE, $F(1,107)=5,31, p=0,02, \eta^{2}$ $=0,05$. Así, el promedio en la variable AAA de los casos de NSE bajo fue significativamente más bajo $(M=2,47, D E=0,50)$ que el de los casos de NSE alto $(M=2,71, D E$ $=0,57), 95 \%$ ICs $[2,34,2,61]$ y $[2,55,2,87]$,

Tabla 2

Media y Desviación Estándar por Dimensión, Según Grupo

\begin{tabular}{lcccccccc}
\hline \multirow{2}{*}{ Dimensiones } & \multicolumn{2}{c}{ Hombre } & \multicolumn{2}{c}{ Mujer } & \multicolumn{2}{c}{ NSE alto } & \multicolumn{2}{c}{ NSE bajo } \\
\cline { 2 - 8 } & $M$ & $D E$ & $M$ & $D E$ & $M$ & $D E$ & $M$ & $D E$ \\
\hline CP & 2,57 & 0,37 & 2,56 & 0,41 & 2,68 & 0,36 & 2,46 & 0,39 \\
AAA & 2,57 & 0,58 & 2,61 & 0,50 & 2,71 & 0,57 & 2,47 & 0,50 \\
EFE & 3,32 & 0,66 & 3,43 & 0,72 & 3,63 & 0,63 & 3,13 & 0,66 \\
\hline
\end{tabular}


respectivamente, demostrando que los profesores en formación esperan que los niños de NSE bajo necesiten con mayor frecuencia AAA. Nuevamente se observa la dinámica anterior, pues los datos parecen no apoyar la hipótesis relativa a la interacción de los factores de NSE y género, pero los estudiantes de pedagogía sí muestran una tendencia a evaluar más negativamente los casos de NSE bajo.

Respecto de la variable EFE, la hipótesis planteada esperaba una interacción de los factores NSE y género, de modo que los participantes expresaran peores expectativas futuras para los casos de NSE bajo y de sexo femenino. Nuevamente se observa la dinámica anterior, no presentándose la interacción esperada, $F(3,107)=0,82, p=$ 0,77 . Pero el análisis de los efectos simples de cada variable mostró que, si bien no se presentan diferencias significativas de los promedios de EFE de acuerdo al género del protagonista de la historia, $F(1,107)=0,71$, $p=0,40$, sí es posible observarlas al comparar los promedios de la variable EFE en los casos de diferente NSE, $F(1,107)=15,66, p$ $<0,001, \eta^{2}=0,13$. Así, la variable EFE en los casos de NSE bajo presentó un promedio significativamente más bajo $(M=3,13$, $D E=0,66)$ que el de los casos de NSE alto $(M=3,63, D E=0,63), 95 \%$ ICs $[2,95,3,31]$ y $[3,46,3,81]$, respectivamente, demostrando que los profesores en formación poseerían peores expectativas de futuro para los alumnos de menor NSE.

Una hipótesis final planteaba que no existen diferencias significativas en las percepciones que, acerca de los estudiantes de las historias, tienen los profesores en formación en el primero o último año de carrera universitaria. Los datos muestran la tendencia contraria: la información apoya la existencia de diferencias significativas de los promedios de las tres variables dependientes entre los profesores en formación en el primer año de estudio y los de cuarto año (CP: $t[106]=-4,46, p<0,001, d=-0,86$; AAA: $t[106]=-3,58, p<0,001, d=-0,69$; EFE: $t[106]=-2,42, p=0,01, d=-0,47)$. Así, para todos los casos, los alumnos de primer año presentaron las percepciones más negativas hacia los estudiantes protagonistas de las historias, a saber, consideraban que los estudiantes de las historias presentaban peores $\mathrm{CP}$, tenían una mayor necesidad de AAA y tenían peores EFE.

Respecto de las interacciones y efectos simples del género y NSE, se pudo observar que la dinámica de resultados es muy similar a la obtenida con el grupo completo de estudiantes. En los alumnos de primer año no se observó una interacción significativa del género y el NSE en ninguna de las variables dependientes, que refleje la tendencia a evaluar más negativamente a los personajes de las historias que son de género femenino y NSE bajo. Un análisis de los efectos simples de cada variable mostró que, respecto del género del protagonista de la historia, parece no haber diferencias significativas en los promedios de CP, AAA y EFE, pero sí respecto del NSE del personaje, pero solo en $\operatorname{CP}$ y $\operatorname{EFE}(F[1,66]=5,55, p$ $=0,02, \eta^{2}=0,08$ y $F[1,66]=6,20, p=0,01$, $\eta^{2}=0,09$, respectivamente) y no en AAA. Esto último refleja una tendencia distinta a la observada en la muestra total. Así, los profesores en formación de primer año de carrera parecen no esperar resultados diferentes para niños y niñas en las variables $\mathrm{CP}$, AAA y EFE, pero esperarían que los niños y niñas de NSE bajo presenten comparativamente peores $\mathrm{CP}(M=2,34, D E=$ $0,39,95 \%$ IC $[2,21,2,48])$ y peores perspectivas de futuro $(M=3,05, D E=0,71,95 \%$ IC $[2,81,3,30])$ que los niños de NSE alto (CP: $M=2,55, D E=0,33,95 \%$ IC $[2,43$, 2,68] y EFE: $M=3,46, D E=0,63,95 \%$ IC $[3,24,3,69])$. Por otra parte, los estudiantes de pedagogía de primer año muestran la tendencia a evaluar de manera similar la necesidad de AAA para los niños de NSE bajo y alto.

Por su parte, el grupo de alumnos de pedagogía de cuarto año presentó una tendencia similar a los del grupo general, a saber, no se observó una interacción significativa entre género y NSE en ninguna de las variables dependientes que demostrara que evalúan más negativamente a los personajes de las historias de sexo femenino y NSE bajo. Asimismo, un análisis de los efectos simples mostró que, respecto del género del protagonista de la historia, no se observan diferencias significativas de los promedios de CP, AAA y EFE, pero sí respecto del NSE 
del personajes para todas las variables dependientes $\left(\mathrm{CP}: F[1,40)=5,00, p=0,03, \eta^{2}\right.$ $=0,11$;AA: $F[1,40]=7,70, p<0,001, \eta^{2}=$ 0,16 y EFE: $F[1,40]=12,91, p<0,001, \eta^{2}$ $=0,25)$. En resumen, los profesores en formación de último año de carrera parecieran no esperar resultados diferentes para niños y niñas en CP, AAA y EFE pero sí que los niños y niñas de NSE bajo presenten comparativamente peores $\mathrm{CP}(M=2,66, D E=$ $0,32,95 \%$ IC $[2,51,2,88])$, mayor necesidad de AAA $(M=2,60, D E=0,50,95 \%$ IC $[2,37$, $2,82])$ y peores perspectivas de futuro $(M=$ $3,26, D E=0,58,95 \%$ IC $[3,00,3,53])$ que los niños de NSE alto (CP: $M=2,88, D E=0,31$, 95\% IC [2,73, 3,02]; AAA: $M=3,06, D E=$ 0,55, 95\% IC [2,79, 3,32] y EFE: $M=3,90$, $D E=0,55,95 \%$ IC $[3,64,4,16])$.

\section{Discusión}

Los resultados de este estudio muestran, en conjunto, que los profesores en formación presentan la tendencia a asignar a los estudiantes de NSE bajo una mayor probabilidad de poseer CP negativas (e.g., baja autoestima, inestable, lento), una mayor probabilidad de necesidad de AAA (e.g., apoyo extra-escolar, necesidad de una intervención especial) y futuros menos promisorios (e.g., mayor probabilidad de abandonar la escuela, mayor probabilidad de repitencia, menor puntaje PSU). Estos hallazgos concuerdan con investigaciones previas (Auwarter \& Aruguete, 2008; Hamilton, Sherman \& Ruvolo, 1990) que mostraron que, a igual rendimiento académico, los niños de NSE alto son juzgados más positivamente que los niños de NSE bajo. Este tipo de razonamiento tiene una importante consecuencia para la labor docente: los profesores que piensan que el NSE es un factor determinante del rendimiento de sus alumnos actuarán en consecuencia y sentirán que tienen menos herramientas para trabajar con este grupo de niños. Este efecto se refleja en una investigación que mostró que el 75\% de los profesores de escuelas que atienden población de bajo NSE presenta un bajo sentido de autoeficacia para enseñar (Warren, 2002).

Los datos reflejan que el género de los personajes no afectó las evaluaciones rea- lizadas por los participantes de la investigación. De este modo, pareciera rechazarse la hipótesis que planteaba que el género femenino de los personajes predispone a evaluaciones más negativas por parte de los estudiantes de pedagogía. Esto es en sí mismo un resultado positivo, puesto que los futuros profesores participantes de esta investigación no estarían asociando al género creencias negativas acerca de las capacidades académicas ni tendrían expectativas más bajas para este grupo. Pero este hallazgo no explicaría por qué las mujeres presentan en Chile un rendimiento académico más pobre que los varones en algunas materias. Al respecto, se puede especular que en el presente estudio, al plantear a los estudiantes de pedagogía un dilema de género, se pudo haber presentado deseabilidad social: pudieron haber contestado más bien lo que pensaban que era correcto, más que lo que verdaderamente creían. Futuras investigaciones y diferentes diseños metodológicos serán necesarios tanto para confirmar esta hipótesis como para profundizar en el conocimiento de las causas de las diferencias en Chile en el logro académico de niños y niñas.

Los datos mostraron una pequeña diferencia en las respuestas de los alumnos de pedagogía de primero y cuarto año. Si bien ambos grupos presentaron de manera consistente expectativas más bajas para los niños de NSE bajo en las variables CP y EFE, los de primer año consideraron que tanto los alumnos de NSE alto y bajo necesitaban de igual AAA. Esto podría explicarse en dos sentidos. El primero es que es más probable que se crea que un niño con problemas de rendimiento necesite de AAA, más allá de su NSE de pertenencia, lo que puede explicar los resultados de los alumnos de primer año. El segundo es que la diferencia de respuesta observada entre los estudiantes de cuarto año en esta variable, de acuerdo al NSE del estudiante hipotético, podría explicarse porque estos estudiantes, al tener ya una inserción más intensa -por sus prácticas profesionales- en el sistema educativo, cuentan con una visión más realista de las verdaderas necesidades de AAA que presentan los niños de NSE bajo, especialmente por la baja calidad académica promedio 
de los establecimientos educacionales a los que asisten. A este respecto, se puede pensar que, a diferencia de las otras dos variables dependientes evaluadas en el presente estudio, la variable AAA parece ser una que en la realidad de Chile está efectivamente relacionada al NSE: los niños de NSE bajo reciben una educación de menor calidad y, por lo tanto, necesitan de un mayor AAA.

Es relevante encontrar hallazgos como los relativos a las variables CP y EFE entre estudiantes de pedagogía. La etapa que conforma la formación pedagógica inicial es el momento clave para abordar temas como el de las expectativas, creencias y estereotipos ya instalados, como es el caso de los observados en estas variables respecto del NSE. Y es así porque dentro de la formación pedagógica generalmente se considera la instancia de las prácticas, en las que de manera gradual los estudiantes de pedagogía se ven inmersos en el sistema escolar, teniendo sus primeras experiencias de contacto con niños y niñas que serán similares a sus futuros alumnos. Asimismo, asociadas a estas prácticas se encuentran también las primeras reflexiones acerca del rol del profesor y acerca de las experiencias que han vivido en las escuelas a las que asisten. Es también en este espacio donde se puede instalar una intervención dirigida a conocer las expectativas y estereotipos que manejan e intentar modificarlas. Al respecto, Good y Nichols (2001) proponen un modelo de intervención dirigido a cambiar las expectativas que los profesores tienen de ciertos grupos de alumnos, entre otras cosas, a través de enseñar a los profesores los fundamentos de la teoría de expectativas y sus posibles efectos.

La posibilidad de una intervención en el área de las expectativas y estereotipos tiene una directa relación con los resultados relativos a las diferencias observadas entre los alumnos de primer y último año de carrera. Pareciera que los estereotipos acerca de quiénes serán mejores o peores alumnos y de sus características ya están instalados antes de siquiera comenzar a estudiar pedagogía. Esto podría explicar por qué los alumnos de primer año manejan una visión general más negativa acerca de los personajes de la historia. Pero, por otro lado, es im- portante recalcar que pareciera que la formación pedagógica inicial algo está haciendo bien en esta área, pues los alumnos de último año manejan un estereotipo menos marcado negativamente acerca de los alumnos que tienen dificultades académicas. Futuras investigaciones debieran enfocarse en esclarecer tanto los estereotipos de entrada de los futuros profesores como la manera en que la formación pedagógica inicial se hace cargo o puede intervenir exitosamente en esta área. Adicionalmente, estos resultados no solo desafían a los programas de formación de profesores, sino que a toda la sociedad, pues son los mensajes de la cultura los que tiñen las expectativas y estereotipos de los jóvenes, en este caso, los estudiantes de pedagogía de primer año.

Una limitación del presente estudio radica en que para identificar al estudiante de la historia como de NSE bajo se utilizaron tanto claves relativas a la inserción profesional de los padres como al lugar donde su familia vivía y el tipo de dependencia de la escuela a la que asistía. Al respecto, en Chile la realidad muestra que masivamente las escuelas municipales, las que se asocian a NSE bajo, presentan en promedio un bajo rendimiento académico (Chile, Ministerio de Educación, 2007). Esto último puede resultar confuso cuando se evalúan las expectativas respecto a AAA y EFE, pues el efecto del NSE se confunde con la mala calidad de las escuelas a las que los niños de NSE bajo asisten. Aun así, si bien este es un problema que se debe corregir en futuras investigaciones, es relevante conocer que los estudiantes de pedagogía parecen comenzar su carrera profesional con expectativas más negativas respecto de los niños de NSE bajo, más allá de que estas se radiquen solo en su NSE o en una combinación de este con las oportunidades que el sistema educacional les ofrecerá.

Una limitación adicional podría eventualmente radicar en que la manera en la que se juzga a un estudiante hipotético no se corresponde necesariamente con lo que sucede en la vida real. Esto último es por ahora una hipótesis que solo investigaciones futuras podrán confirmar.

Por último, un estudio que utilice una muestra probabilística que contenga alum- 
nos de diversos programas de formación de profesores podría permitir una mayor generalización de los hallazgos encontrados.

\section{Referencias}

Auwarter, A. E. \& Aruguete, M. S. (2008). Effects of student gender and socioeconomic status on teacher perceptions. The Journal of Educational Research, 101, 243-246. doi:10.3200/JOER.101.4.243-246

Chile, Ministerio de Educación (2007). PISA 2006: rendimientos de estudiantes de 15 años en ciencias, lectura y matemática. Santiago, Chile: Autor, Unidad de Curriculum y Evaluación. Extraído de http://www.simce.cl/fileadmin/Documentos_y_ archivos_SIMCE/PISA2006/PISA_2006.pdf

Chile, Sistema de Medición de Calidad de la Educación (2008). Resultados nacionales SIMCE 2007. Santiago, Chile: Autor. Extraído de http:// mt.educarchile.cl/MT/jjbrunner/archives/libros/ SIMCE/SIMCE_2007.pdf

Chile, Sistema de Medición de Calidad de la Educación (2009). Resultados nacionales SIMCE 2008. Santiago, Chile: Autor. Extraído de http://www. simce.cl/fileadmin/Documentos_y_archivos_ SIMCE/Informes_Resultados_2008/Informe_ Nacional_2008.pdf

Chile, Sistema de Medición de Calidad de la Educación (2010). Resultados nacionales SIMCE 2009. Santiago, Chile: Autor. Extraído de http://www. simce.cl/fileadmin/Documentos_y_archivos_ SIMCE/Informes_Resultados_2009/Informe_ Nacional_2009.pdf

Derman-Sparks, L. \& The A.B.C. Task Force (1989). Anti-bias curriculum: Tools for empowering young children (NAEYC $\mathrm{N}^{\circ}$ 242). Washington, DC: National Association for the Education of Young Children.

Diamond, J. B., Randolph, A. \& Spillane, J. P. (2004). Teachers' expectations and sense of responsibility for students learning: The importance of race, class, and organizational habitus. Anthropology \& Education Quarterly, 35, 75-98. doi:10.1525/ aeq.2004.35.1.75

Dusek, J. B., \& Joseph, G. (1983). The bases of teacher expectancies: A meta-analysis. Journal of Educational Psychology, 75, 327-346. doi:10.1037/0022-0663.75.3.327

Good, T. L. \& Nichols, S. L. (2001). Expectancy effects in the classroom: A special focus on improving the reading performance of minority students in firstgrade classrooms. Educational Psychologist, 36, 113-126. doi:10.1207/S15326985EP3602_6

Gray, C. \& Leith, H. (2004) Perpetuating gender stereotypes in the classroom: A teacher perspective. Educational Studies, 30, 3-17. doi:10.1080/03055 69032000159705

Fecha de recepción: Diciembre de 2009.

Fecha de aceptación: Agosto de 2010.
Hamilton, D. L., Sherman, S. J. \& Ruvolo, C. M. (1990). Stereotype-based expectancies: Effects on information processing and social behavior. Journal of Social Issues, 46(2), 35-60. doi:10.1111/ j.1540-4560.1990.tb01922.x

Jussim, L. \& Harber, K. D. (2005). Teacher expectations and self-fulfilling prophecies: Knows and unknowns, resolved and unresolved controversies. Personality and Social Psychology Review, 9, 131155. doi:10.1207/s15327957pspr0902_3

Ladd, J. A. \& Linderholm, T. (2008). A consequence of school grade labels: Preservice teachers' interpretations and recall of children's classroom behavior. Social Psychology of Education, 11, 229-241. doi:10.1007/s11218-007-9049-4

MIDE UC (2008). Particularidades de Chile en los resultados PISA 2006. Santiago, Chile: Pontificia Universidad Católica de Chile. Extraído de http:// www.mideuc.cl/docs/PisaMide.PDF

Mistry, R. S., White, E. S., Benner, A. D \& Huynh, V. W. (2009). A longitudinal study of the simultaneous influence of mothers' and teachers' educational expectations on low income youth's academic achievement. Journal of Youth and Adolescence, 38, 826-838. doi:10.1007/s10964-008-9300-0

Navas, L. \& Sánchez, A. (2010). Actitudes de los estudiantes de pedagogía de las regiones del Bío Bío y la Araucanía de Chile hacia la presencia de niños inmigrantes en la escuela: análisis diferenciales. Psykhe, 19(1), 47-60. doi:10.4067/ S0718-22282010000100004

Palardy, G. J. \& Rumberger, R. W. (2008). Teacher effectiveness in first grade: The importance of background qualifications, attitudes, and instructional practices for student learning. Educational Evaluation and Policy Analysis, 30, 111-140. doi: $10.3102 / 0162373708317680$

Rashid, H. M. (2009). From brilliant baby to child placed at risk: The perilous path of African American boys in early childhood education. The Journal of Negro Education, 78, 347-358. Extraído de http://proquest.umi.com.ezproxy.puc. $\mathrm{cl} /$ pqdweb?did=1902450141\&sid=6\&Fmt=3\&clie ntId $=52738 \&$ RQT $=309 \&$ VName $=$ PQD

Rosenthal, R. \& Jacobson, L. (1966). Teachers' expectancies: Determinants of pupils' IQ gains. Psychological Reports, 19, 115-118. doi:10.2466/ PR0.19.5.115-118

Warren, S. R. (2002). Stories from the classroom: How expectations and efficacy of diverse teachers affect the academic performance of children in poor urban schools. Educational Horizons, 80, 109116. Extraído de http://vnweb.hwwilsonweb.com. ezproxy.puc.cl/hww/jumpstart.jhtml?recid=0bc05 f7a67b1790ed51a56931801d424ba96f3d3be1b3c d7d6e1f7c6a65a9c0b5f9e340f0c765474\&fmt=P 